\title{
EXTRAÇÃO DE ROTENONA COM FLUIDO EM ESTADO SUPERCRÍTICO DE DERRIS ELLIPTICA E TEPHROSIA CANDIDA EM ESCALA INDUSTRIAL
}

\author{
A. MARANGONI ${ }^{1}$, A. BOLZAN ${ }^{2}$, M. ARAUCO LIVIA ${ }^{3}$ \\ 1,2,3 Universidade Federal de Santa Catarina, Departamento de Engenharia Química e Engenharia de Alimentos \\ E-mail para contato: alessandramarangon@yahoo.com.br
}

RESUMO -A busca por práticas agrícolas sustentáveis que viabilizem a produção de alimentos seguros e sem contaminantes está entre os desafios atuais do agronegócio. A necessidade de métodos mais eficientes e menos agressivos ao ambiente tem estimulado a pesquisa de novos inseticidas. Neste trabalho desenvolveu-se um processo de obtenção de rotenona em escala industrial que utiliza o $\mathrm{CO}_{2}$ em estado supercrítico com etanol como solvente. Testou-se duas espécies vegetais: A Derris elliptica e Tephrosia candida. Realizou-se testes laboratoriais com extratores de 0,175 litro para a definição dos parâmetros a serem utilizados na escala industrial. Os testes para ampliação de escala foram realizados em unidade industrial composta de dois extratores de 30 litros. O critério de desempenho utilizado foi o rendimento de extrato e a concentração de rotenona. Os resultados indicaram que a espécie vegetal Derris elliptica apresentou o melhor resultado com rendimento de $4,18 \%$ (g/g) de extrato e $31,18 \%$ de rotenona, a 100 bar e $45^{\circ} \mathrm{C}$, com etanol $92,8^{\circ} \mathrm{GL}$.

\section{INTRODUÇÃO}

A produção agrícola tem evoluído de forma significativa neste século. Os avanços da ciência têm fundamentado muitas tecnologias, permitindo a alta produtividade do setor. $\mathrm{O}$ aumento da produção tem provocado um desequilíbrio que compromete o processo produtivo. Para controlar as pragas, diversas substâncias sintéticas vêm sendo utilizadas o que causa resistência nos insetos e geram desequilíbrios ecológicos e agrava os problemas que deveriam combater. Assim, a procura por um inseticida de largo espectro e com rápida degradação, sem causar impacto ambiental, tem sido uma preocupação permanente.

O uso de inseticidas botânicos no controle de pragas ainda apresenta algumas limitações. A disponibilidade de matérias-primas para extração dos compostos e a dificuldade na padronização dos extratos são fatores que dificultam o uso desses produtos em agricultura de grande escala. Também há limitações relacionadas às técnicas de extrações das moléculas ativas.

A extração dos inseticidas botânicos tem sido pouco estudada e as metodologias desenvolvidas são em escala laboratorial. A viabilidade econômica da produção industrial desses inseticidas pode ser uma alternativa sustentável ao controle de pragas sem o uso dos inseticidas organossintéticos.

Rotenona e os rotenóides são isoflavonóides produzidos em raízes e rizomas de algumas plantas tropicais dos gêneros Derris, Lonchocarpus e Tephrosia, (Xiao-Jun et al, 2009). O inseticida rotenona tem a sua molécula registrada na American Chemical Society (cas), como: 83-79-4. Apresenta peso molecular de 394,42 u, com classificação toxicológica de grau III. Sua 
fórmula química é $\mathrm{C}_{23} \mathrm{H}_{22} \mathrm{O}_{6}$. A rotenona é rapidamente degradada pela luz, não gerando resíduos nas folhas das plantas após a aplicação. Em contato com a água, degrada-se rapidamente (Extoxnet, 1996).

Os extratos botânicos normalmente são extraídos com solventes orgânicos. A importância crescente por inseticidas botânicos criou demanda pelo desenvolvimento de novas técnicas que não agridam o meio ambiente e nem contamine um produto orgânico com solventes. A extração com dióxido de carbono $\left(\mathrm{CO}_{2}\right)$ supercrítico é um processo tecnicamente viável para obtenção de inseticidas orgânicos livre de solventes.

A densidade relativamente alta dos fluidos supercríticos faz com que possuam um poder de solvatação semelhante aos líquidos e maior em relação aos gases. A principal vantagem dos fluidos supercríticos em relação aos líquidos é que, por apresentarem uma viscosidade menor e um coeficiente de difusividade de massa maior do que no estado líquido, associado com altas densidades, faz com que esses fluidos possuam uma maior capacidade para penetrar nas matrizes porosas, das quais se extraem as moléculas bioativas (Imison \& Unthank, 2000). Para melhorar o processo de extração de compostos polares, além do uso do $\mathrm{CO}_{2}$ supercrítico, diversos estudos utilizam outros compostos como cossolvente. Este trabalho apresenta os resultados do desenvolvimento do processo de extração de rotenona com $\mathrm{CO}_{2}$ em estado supercrítico puro e com etanol de dois gêneros diferentes: Derris e Tephorosia.

\section{2- MATERIAL E METODOS}

Os experimentos realizados neste trabalho foram desenvolvidos em duas etapas. $\mathrm{Na}$ primeira, realizou-se um estudo na planta piloto do Laboratório de Controle de Processos (LCP) do Departamento de Engenharia Química e Engenharia de Alimentos da Universidade Federal de Santa Catarina (LCP/ENQ/UFSC). Realizou-se a segunda em um equipamento industrial com dois extratores de 30 litros, na empresa Technessentia Compostos Naturais Ltda.

Neste trabalho, utilizaram-se duas espécies vegetais, Derris elliptica e Tephrosia candida. Para a Derris elliptica, utilizou-se raízes de plantas coletadas na Amazônia. A Tephrosia candida utilizada foi coletada na região de Ratones, Florianópolis, SC. Utilizaram-se as folhas e galhos, secas em estufa com circulação e renovação de ar por 24 a 30 horas a uma temperatura de $30^{\circ} \mathrm{C}$.

Para as duas espécies vegetais o procedimento de classificação e determinação dos parâmetros foi mesmo. Na determinação do teor de umidade, dispuseram as amostras de planta em estufa a $105^{\circ} \mathrm{C} \pm 1{ }^{\circ} \mathrm{C}$, até peso constante. A umidade inicial da planta foi de $20 \%$ e a final de $9 \% \pm 0,5 \%$. Para determinar a distribuição de tamanho de partículas (DTP), classificaram-se as partes das plantas moídas por um conjunto de doze peneiras da série Tyler, com abertura entre 0,000 e 2,362 mm. O material retido em cada peneira foi pesado em balança analítica com precisão de 0,001g. Selecionou-se a amostra de malha Tyler 32, com diâmetro médio de 0,598 $\mathrm{mm}$.

O volume interno do extrator da escala laboratorial é de 0,175 litro e o extrator de escala industrial é composto por dois extratores de 30 litros. Em ambas extrações utilizou-se $\mathrm{CO}_{2}$ com $99,95 \%$ de pureza e etanol $92,8^{\circ} \mathrm{GL}$. O tempo total de extração foi 300 minutos, com 60 minutos de período estático. A vazão do $\mathrm{CO}_{2}$ supercrítico foi mantida em 3,0g/min para todas as condições experimentais testas. 
$\mathrm{Na}$ unidade em escala laboratorial realizaram-se os experimentos para determinar as condições que se obtém maior rendimento de extrato e de rotenona.

Os equipamentos para extração a alta pressão em escala laboratorial esta esquematizado na Figura-1.

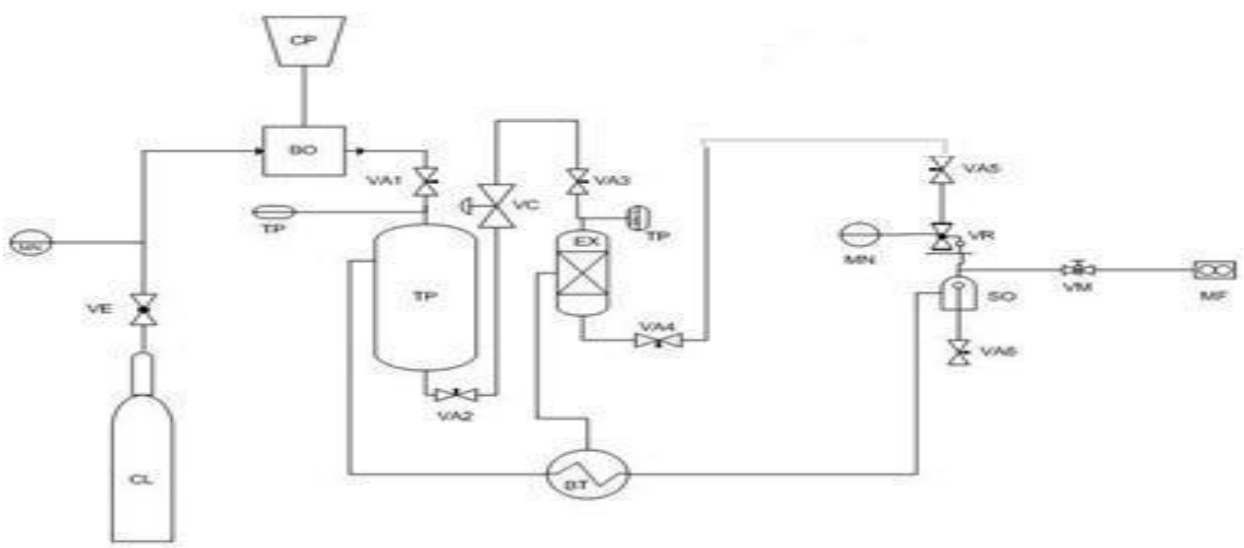

Figura-1. Layout da Planta de Extração Supercrítica em escala de laboratório

$\mathrm{BC}-$ Banho termocriostático

BO - Booster

MF - Medidor de fluxo

$\mathrm{TP}$ - Transdutores de pressão

VR - Válvula redutora de pressão
BT - Banho termostático

CL- Cilindro de $\mathrm{CO}_{2}$

MN - Manômetros

SO- Separador de óleos.

VE - Válvula de esfera

VC - Válvula de controle
CP- Compressor de ar

EX - Extrator

TP- Tanque pulmão.

VA - Válvula de agulha

VM - Válvula micrométrica

Na tabela-1 estão listadas as condições operacionais dos experimentos realizados no laboratório. Todos os experimentos foram realizados em duplicatas.

Tabela-1. Condições experimentais dos ensaios realizados em laboratório.

\begin{tabular}{|c|c|c|c|}
\hline Pressão (bar) & Temperatura $\left({ }^{\circ} \mathbf{C}\right)$ & Cossolvente $(\%)$ & Análises realizadas \\
\hline $\mathbf{1 0 0}$ & 45,50 e 60 & sem & Rendimento e cromatografia \\
\hline $\mathbf{1 0 0}$ & 45,50 e 60 & 180 & Rendimento e cromatografia \\
\hline $\mathbf{1 0 0}$ & 65 e 70 & 180 & Rendimento e cromatografia \\
\hline
\end{tabular}

No equipamento industrial realizaram-se experimentos para se avaliar os efeitos da ampliação de escala. A figura-2 mostra o layout da unidade industrial. 


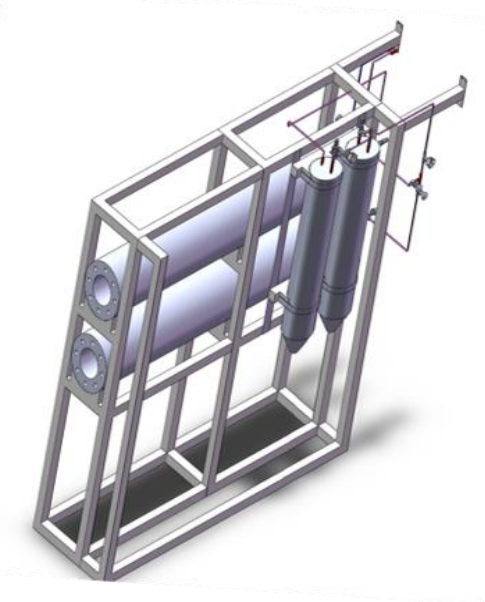

Figura-2. Layout da Planta de Extração Supercrítica em escala industrial.

Para realização desses experimentos utilizou-se as melhores condições experimentais obtidas no estudo realizado no laboratório. O parâmetro que definiu essa escolha foi a maior concentração de rotenona no extrato obtido. Na tabela-2 estão especificadas as condições dos testes realizados em escala industrial.

Tabela-2. Condições experimentais dos ensaios realizados na escala industrial

\begin{tabular}{|c|c|c|c|c|}
\hline Pressão (bar) & Temperatura $\left({ }^{\circ} \mathrm{C}\right)$ & Cossolvente $\%$ & Pressurização & Análises realizadas \\
\hline 100 & 55 & 180 & 1 & Rendimento e cromatografia \\
\hline 100 & 60 & 180 & 1 & Rendimento e cromatografia \\
\hline 100 & 70 & 180 & 1 & Rendimento e cromatografia \\
\hline 100 & 60 & 180 & 2 & Rendimento e cromatografia \\
\hline
\end{tabular}

Para quantificar a rotenona utilizou-se um equipamento HPLC, com coluna Supelcosil LC-18, com tamanho de partícula de $5 \mu \mathrm{m}$, diâmetro interno de 4,6 mm e tamanho de coluna 25 $\mathrm{cm}$. A fase móvel foi uma solução de metanol/água (80:20) e detector UV-Vísivel em $254 \mathrm{~nm}$.

\section{3-RESULTADOS E DISCUSSÕES}

Determinou-se o rendimento do processo calculando-se a relação entre a massa extraída e massa total presente no extrator.

Primeiramente, determinou-se quais são os parâmetros operacionais que resultaram na obtenção de extratos com os maiores rendimento em massa e concentração de rotenona. Utilizaram-se os parâmetros obtidos como base para os testes de ampliação de escala.

É importante observar que as pressões utilizadas nos experimentos não foram superiores a 100 bar devido às limitações operacionais do equipamento industrial.

Os resultados obtidos nos primeiros experimentos estão descritos na tabela-3. É importante ressaltar que os valores de rendimentos calculados são valores médios das duplicatas das extrações. Uma das características importantes da extração com $\mathrm{CO}_{2}$ supercrítico é a capacidade em dissolver compostos orgânicos de baixa volatilidade. Essa propriedade é 
diretamente proporcional a alta densidade do solvente (REVERCHON \& MARCO, 2006). A definição da pressão e temperatura de extração é um fator importante que afeta a composição final do extrato e o rendimento do processo (MUKHOPADHYAY, 2000). A condição operacional que obteve o melhor rendimento para as duas espécies foi de $100 \mathrm{bar}$ com $45^{\circ} \mathrm{C}$, que é o experimento em que o $\mathrm{CO}_{2}$ possui a maior densidade, conforme mostra a tabela-3. Os resultados obtidos nos testes realizados comprovaram as afirmações de diversos pesquisadores em relação à solubilização do solvente e sua relação com a densidade, ou seja, o aumento isotérmico da pressão aumenta a densidade do solvente, que, consequentemente, aumenta a solubilidade do soluto, o que resulta em um maior rendimento da extração (BRUNNER, 1994, DEL VALLE \& AGUILERA, 1988). Também pode se observar que, como a pressão utilizada neste trabalho é relativamente baixa, $100 \mathrm{bar}$, quando se utilizaram temperaturas maiores de $55^{\circ} \mathrm{C}$ houve uma diminuição da densidade do $\mathrm{CO}_{2}$. $\mathrm{O}$ aumento da temperatura causa aumento na massa específica do solvente quando estão associadas a elevadas pressões. Quando se utiliza pressões mais baixas, o aumento da temperatura diminui a densidade do $\mathrm{CO}_{2}$ (TAYLOR, 1996; BRUNNER, 1994). A análise dos resultados obtidos nesta fase experimental mostraram que a maior taxa de extração se deve à capacidade do $\mathrm{CO}_{2}$ de dissolver os compostos a serem extraídos e que se encontram disponíveis na superfície do vegetal e ao maior poder de penetração nos poros do leito e da matriz vegetal. A figura-3 mostra o comportamento dinâmico do processo de extração, muito semelhante para as duas espécies de vegetal utilizadas.

Tabela-3. Rendimento de extrato de Derris elliptica e Tephrosia cândida e massa específica do $\mathrm{CO}_{2}$ nas extrações com $\mathrm{CO}_{2}$ supercrítico, unidade de laboratório.

\begin{tabular}{|c|c|c|c|c|}
\hline $\begin{array}{c}\text { Temperatura } \\
\left({ }^{\mathbf{}} \mathbf{C}\right)\end{array}$ & $\begin{array}{c}\text { Pressão } \\
\text { (bar) }\end{array}$ & $\begin{array}{c}\text { Massa específica } \\
\left(\mathbf{K g} / \mathbf{m}^{\mathbf{3}}\right)\end{array}$ & $\begin{array}{c}\text { Rendimento (\%) } \\
\text { Extrato e rotenona } \\
\text { Derris Elliptica }\end{array}$ & $\begin{array}{c}\text { Rendimento (\%) } \\
\text { Extrato e rotenona } \\
\text { Tephorisa Cândida }\end{array}$ \\
\hline 45 & 100 & $\mathbf{4 6 1 , 3 1}$ & $\mathbf{1 , 0 9}-\mathbf{8 , 4 1}$ & $\mathbf{1 , 1 4 - 5 , 6 9}$ \\
\hline 50 & 100 & 376,52 & 0,81 & 0,92 \\
\hline 60 & 100 & 292,40 & 0,72 & 0,69 \\
\hline
\end{tabular}

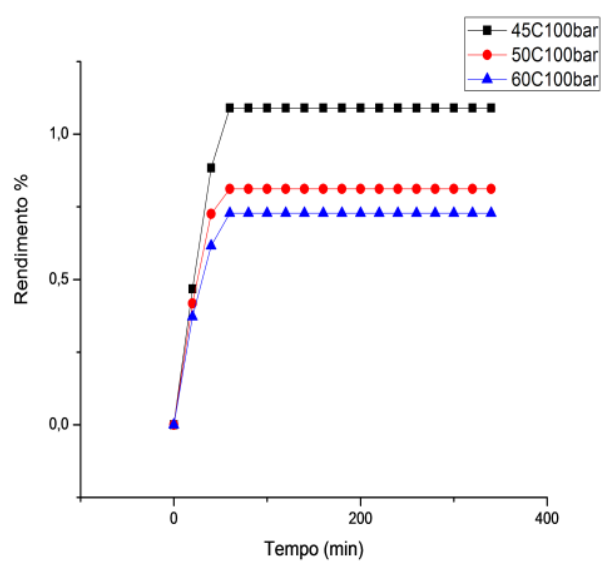

(a)

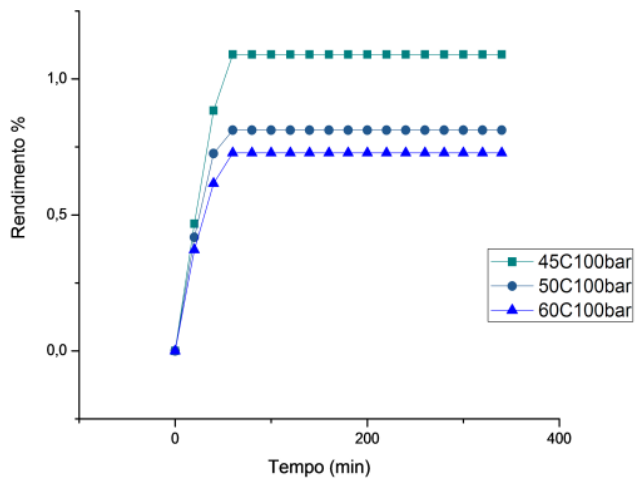

(b)

Figura 3- Curvas de rendimento das extrações por extração supercrítica com 100 bar e em diferentes condições de temperatura na unidade do laboratório. (a) de Derris elliptica e (b) Tephrosia cândida 
A capacidade de extração é diretamente proporcional ao aumento da densidade do $\mathrm{CO}_{2} \mathrm{e}$ ao poder de solubilização do $\mathrm{CO}_{2}$ supercrítico, que depende basicamente da sua densidade. Como regra, reafirma-se que um aumento na densidade do fluido supercrítico implica em um aumento na sua capacidade de solubilizar o soluto (DEL VALLE \& AGUILERA, 1988).

Os resultados obtidos neste experimento são semelhantes aos resultados encontrados por ANDREA et al, (2007), com pressão de 90 bar e $40{ }^{\circ} \mathrm{C}$, que obtiveram o rendimento de $1,2 \%$ com a espécie Derris elliptica.

$\mathrm{O}$ rendimento obtido nas extrações com $\mathrm{CO}_{2}$ supercrítico puro foi relativamente baixo. A rotenona é uma molécula polar, o $\mathrm{CO}_{2}$ puro é apolar, o que resulta numa pouca afinidade pelo composto a ser extraído. Para melhorar a eficiência do processo de extração de compostos polares com o uso de $\mathrm{CO}_{2}$ supercrítico utilizou-se o etanol como cossolvente.

As curvas de rendimento das extrações com etanol estão apresentados na figuras-5. A tabela-4 mostra o rendimento de rotenona para estes experimentos. Para Derris elliptica, a condição operacional que apresentou o rendimento de 5,50\% foi 100 bar e $55^{0} \mathrm{C}$. Para a Tephrosia candida o rendimento de $5,79 \%$ foi obtido com 100 bar e $65^{\circ} \mathrm{C}$. Todas as curvas de extração, para ambas as espécies, apresentaram um comportamento típico de curva de extração com $\mathrm{CO}_{2}$ puro.

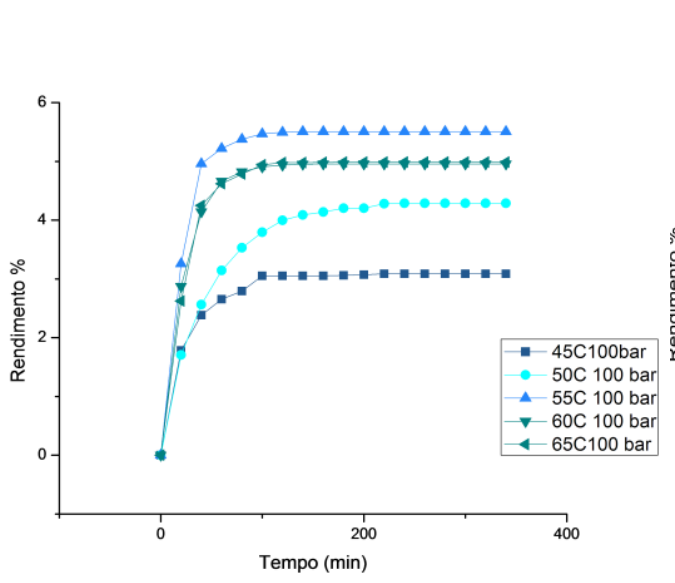

(a)

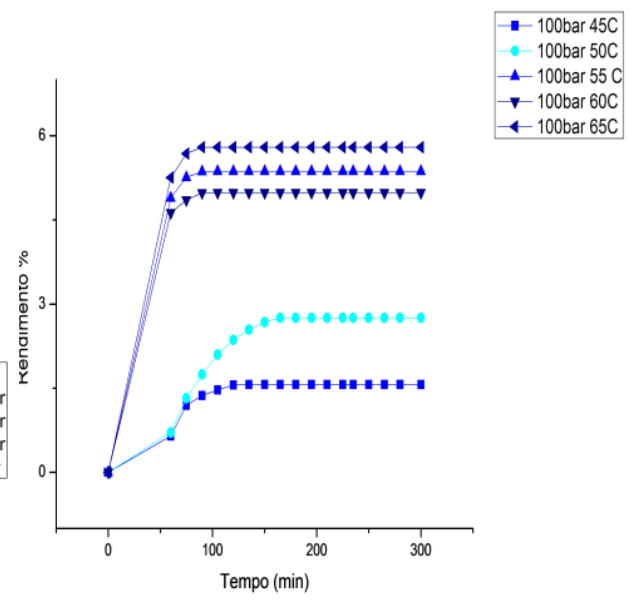

(b)

Figura-5. Curvas de rendimento das extrações de Derris elliptica com cossolvente, utilizando 100 bar e em diferentes condições de temperatura unidade do laboratório. (a) de Derris elliptica e (b) Tephrosia cândida

O uso de cossolvente eleva a densidade da mistura, o que aumenta as interações físicas dipolo/dipolo, dipolo/dipolo induzido e dipolo induzido/dipolo induzido e, consequentemente, o poder de extração e solvatação do $\mathrm{CO}_{2}$ supercrítico. As interações químicas também aumentam com o uso de cossolvente, pois as moléculas dos alcoóis utilizados podem formar ligações como as pontes de hidrogênio (KOPACK, 2003). Os cossolventes aumentam o rendimento e altera a seletividade do processo com a mudança das características do solvente. A polaridade e 
interações específicas com o soluto, como pontes de hidrogênio, favorece a interação com sítios ativos da matriz sólida, a qual aumenta a capacidade do $\mathrm{CO}_{2}$ de extrair eficientemente os compostos desejados (DÍAZ-REINOSO et al.2006; TONTHUBTHIMTHONG et al. 2004).

Tabela-4. Rendimento de extrato e de rotenona de Derris elliptica e Tephrosia candida em diferentes condições experimentais com $\mathrm{CO}_{2}$ supercrítico com etanol, unidade de laboratório.

\begin{tabular}{|c|c|c|c|}
\hline Temperatura $\left({ }^{\circ} \mathrm{C}\right)$ & Pressão (bar) & $\begin{array}{c}\text { Rendimento (\%) Derris elliptica } \\
\text { Extrato e rotenona }\end{array}$ & $\begin{array}{c}\text { Rendimento (\%) Tephrosia cândida } \\
\text { Extrato e rotenona }\end{array}$ \\
\hline 45 & 100 & $3,08(23,84)$ & $1,56(16,70)$ \\
\hline 50 & 100 & $4,28(33,26)$ & $4,98(14,80)$ \\
\hline 55 & 100 & $\mathbf{5 , 5 0 ( 4 2 , 6 1 )}$ & $5,36(14,81)$ \\
\hline 60 & 100 & $4,95(39,17)$ & $2,75(14,12)$ \\
\hline 65 & 100 & $4,98(39,17)$ & $5,79(12,75)$ \\
\hline
\end{tabular}

A partir dos dados experimentais em escala laboratorial pode-se concluir que a espécie que apresentou o maior rendimento de extrato e com uma maior concentração de rotenona foi a espécie Derris elliptica. Também se pode concluir que o melhor rendimento foi obtido com $100 \mathrm{bar}, 55^{\circ} \mathrm{C}$ e $180 \%$ de etanol em massa como cossolvente.

Para os testes de ampliação de escala, realizou-se duas sequências de dez experimentos na unidade industrial com a espécie Derris elliptica. Os testes foram realizados nas seguintes condições: $180 \%$ de etanol em massa como cossolvente, 100 bar, $60^{\circ} \mathrm{C}$, período estático de 60 minutos e vazão de $\mathrm{CO}_{2}$ de $3,00 \mathrm{~g} / \mathrm{min}$. Obteve-se rendimentos médios de 2,53\% e 2,75\%. A ampliação de escala, com o aumento significativo das dimensões da unidade experimental introduziu restrição à transferência de massa não encontradas no equipamento de laboratório. Assimetrias do leito, resultantes da dificuldade de compactação encontradas na escala industrial é a principal causa da diferença de rendimento entre as duas escalas. A existência de caminhos preferencias, originados pela heterogeneidade do leito, fez que partes da matriz vegetal não entrasse em contato com o $\mathrm{CO}_{2}$ e com o etanol. Esse fenômeno reflete-se no cálculo do rendimento, quando a massa extraída será dividida pela massa total presente no extrator e não pela massa de vegetal que realmente entrou em contato com o solvente, resultando e uma eficiência menor para o processo de extração.

Uma maneira encontrada para compensar os efeitos da não homogeneidade do leito foi realizar o processo de extração em dois ciclos. Assim, com duas pressurizações realizadas em sequência e também com 10 repetições e nas mesmas condições operacionais das anteriores, obteve-se um rendimento médio de $4,18 \%$. O efeito de se pressurizar o leito para a primeira extração com a consequente despressurização para a retirado do solvente proporcionou uma movimentação da massa vegetal que determinou uma compactação mais homogênea do leito. A segunda pressurização em sequência poder atingir regiões do leito que não haviam sido atingidas anteriormente, com isso, aumentou-se a eficiência do processo de extração.

O rendimento de $4,18 \%$ com $31,18 \%$ de rotenoma obtidos com as duas pressurizações em sequência determinou um nível de rendimento economicamente viável para uma operação industrial. 


\section{4-CONCLUSÕES}

Com os resultados experimentais obtidos pode-se concluir que a Derris elliptica é a espécie que apresentou o maior rendimento e porcentagem de rotenona para extração com $\mathrm{CO}_{2}$ em estado supercrítico com etanol como cossolvente. O melhor rendimento foi de 4,18\% de extrato, com $31,18 \%$ de rotenona utilizando-se etanol com cossolvente, com duas pressurização do extrator na unidade industrial com dois vasos de 30 litros. A temperatura de $60^{\circ} \mathrm{C}$ e pressão de 100 bar com $180 \%$ de etanol em massa foram as condições operacionais que resultaram os melhores rendimentos.

\section{5-REFERÊNCIAS}

ANDREA,A.D.; ALIBONI,A.; SANTIS,A.D; MARIANI,S.; GORGOGLIONE,D. and RITIENI,A. SFE of Derris elliptica (Wallich) Benth. Roots: Influence of process parameters on yield and purity of rotenone. J. Supercritical Fluids 42 (2007).330-333.

BRUNNER, G., Gas extraction: an introduction to fundamentals of supercritical fluids and the application to separation processes. Steinkopff, 1994. (Topics in physical chemistry). ISBN 9783798509443. Disponível em: <http://books.google-.com.br/books?id=Ocl vAAAAMAAJ>. Citado na página 138.

DEL VALLE, J.M., and J.M. AGUILERA, An Improved Equation for Predicting the Solubility of Vegetable Oils in Supercritical CO , Ibid. 27:1551-1553 (1988).

DIAZ-REINOSO, B. MOURE, A., DOMINGUEZ, H. and PARAJÓ, J.C. (2006). Supercritical CO2 extraction and purification of compounds with antioxidant activity. J. Agric. Food Chem., 54(7):2441-2469.

Extoxnet. Rotenone Pesticide Information Profile. 1996.

http://extoxnet.orst.edu/pips/rotenone.htm

IMISON, B.; UNTHANK, D. Essential oils and other natural ingredients. Rural Industries Research \& Development Corporation, 2000.

MUKHOPADHYAY, M. Natural extracts using supercritical carbon dioxide. USA: CRC Press, 2000.

REVERCHON, E.; DE MARCO, I., Supercritical fluid extraction and fractionation of natural matter. Journal of Supercritical Fluids, v. 38, p. 146-166, 2006.

TAYLOR, L. T. Supercritical Fluid Extraction. New York: Wiley-Interscience Publication, 1996. $181 \mathrm{p}$.

TONTHUBTHIMTHONG P.; DOUGLAS P L; DOUGLAS, S., Extraction of Nimbin from Neem Seeds Using Supercritical CO2 and a Supercritical CO2 methanol Mixture. J. Supercrit. Fluids, vol 30 pp.287-301, 2004.

XIAO-JUN,C.; HAN-HONG,X.; WEN,Y.; AND SHUN-ZI,L., Research on the effect of photoprotectants on photostabilization of rotenone. Journal of Photochemistry and Photobiology B: Biology. 95 p.93-100.2009. 